\title{
EXPERIENCIAS Y RESULTADOS DE INVESTIGACIÓN PARTICIPATIVA TERRITORIAL: LA MESA DE TRABAJO DEL BARRIO NUEVA CHUBUT, PUERTO MADRYN (ARGENTINA)
}

\section{EXPERIENCES AND RESULTS OF TERRITORIAL PARTICIPATORY RESEARCH: THE WORKING TABLE OF NUEVA CHUBUT NEIGHBORHOOD, PUERTO MADRYN (ARGENTINA)}

\author{
Paula Ferrari ${ }^{1}$ \\ 1 Universidad Nacional de la Patagonia (UNP), Chubut, Argentina
}

Correspondência para: Paula Ferrari (mapaulaferrari@yahoo.com.ar)

doi: 10.12957/geouerj.2019.44831

Recebido em: 27 ago. 2019 | Aceito em: 17 set. 2019

\section{RESUMEN}

El artículo presenta la experiencia de conformación y los primeros resultados de la mesa de trabajo del Barrio Nueva Chubut en Puerto Madryn (Patagonia Argentina). El barrio nace a partir de la toma de tierras en el año 2003 por parte de familias que ante la necesidad de acceso al hábitat vieron restringidas sus posibilidades mediante los mecanismos formales de acceso al mismo. Reconociendo el valor del diálogo de saberes, el trabajo se aborda aplicando metodologías participativas de investigación en el territorio, con el objetivo de hacer conversar el saber científico con el saber de los actores comunitarios. La tarea de campo iniciada en 2015 permitió consolidar los lazos necesarios con los actores y constituir la mesa de trabajo. Se desarrollan las acciones realizadas desde la llegada al territorio y las diversas estrategias de diagnóstico y de trabajo comunitario empleadas, basadas en la experiencia de investigación participativa.

Palavras-chave: participación. actores. territorio. acceso al hábitat. Puerto Madryn.

\begin{abstract}
The article presents the experience of conformation and first results of the working table of Nueva Chubut Neighborhood in Puerto Madryn (Patagonia Argentina). The neighborhood was born from taking of land in 2003 by families that faced the need to access the habitat were restricted their possibilities through formal mechanisms of access to it. Recognizing the value of the dialogue of knowledge, the work applies participatory research methodologies, with the aim of making scientific knowledge talk with the knowledge of the actors in the territory. The field tasks initiated in 2015 allowed consolidate the necessary links with actors and begin to constitute the work table. The actions carried out since the arrival to the territory consisted in diagnostic and community work, based on the experience of participatory research.
\end{abstract}

Keywords: participation. actors. territory. habitat access. Puerto Madryn.

\section{INTRODUCCIÓN}

Desde el proyecto de investigación "Procesos de Transformación con Inteligencia Territorial: Configuraciones urbanas a partir de la Toma de Tierras en Puerto Madryn (Chubut)" desarrollado en el ámbito del Consejo Nacional de Investigaciones Científicas y Técnicas ${ }^{1}$, se propone como objetivo

\footnotetext{
${ }^{1}$ En adelante CONICET-Argentina.

(c) 2019 Ferrari. Este é um artigo de acesso aberto distribuído sob os termos da Licença Creative Commons Atribuição-Não ComercialCompartilha Igual (CC BY-NC-SA 4.0), que permite uso, distribuição e reprodução para fins não comercias, com a citação dos autores e da fonte original e sob a mesma licença.
} 
general elaborar un diagnóstico integral de las problemáticas territoriales y ambientales derivadas de los procesos de toma de tierras en el sector noroeste de la ciudad de Puerto Madryn, ubicada en la región Patagónica Argentina. En el marco del diagnóstico se proyecta concretar una agenda de Intervención y Transformación con los diversos actores presentes, que dé respuestas a los problemas identificados y percibidos por la comunidad. Desde el mencionado contexto investigativo surgen los resultados presentados en este trabajo, vinculados a la trasmisión de experiencias y primeros resultados del proceso de investigación-acción participativa derivados de la Mesa de Trabajo del Barrio Nueva Chubut. La Mesa de Trabajo se constituye en un mecanismo y/o instrumento que reúne o convoca a diversos actores (públicos, privados, académico-científicos, organizaciones de la sociedad civil) en un momento y espacio, con el objetivo de acercar miradas, opiniones y participaciones sobre problemáticas territoriales de una comunidad determinada. Su propósito es vehiculizar procesos de gestión compartida del territorio, mediante el establecimiento de la vinculación y comunicación entre los actores en el territorio y la posibilidad de concretar reuniones de trabajo permanente.

La labor de campo iniciada en el año 2015 permitió tomar contacto con los actores presentes en el barrio e ir construyendo lazos con ellos y con diversas asociaciones y organizaciones, con quienes se organizaron diversos encuentros y reuniones tendientes a constituir una Mesa de Trabajo Permanente orientada a identificar las necesidades prioritarias, y a la vez a fortalecer y promover procesos de participación ciudadana y a consolidar el accionar de las asociaciones vecinales y comunitarias potenciando la confianza y el vínculo con los/as vecinos/as. Hacia el mes de octubre de 2018 se inició el trabajo con la referente del barrio, algunos vecinos y vecinas apuntando al fortalecimiento de la Mesa de Trabajo como espacio de encuentro común y de desarrollo de actividades comunitarias. En el transcurso de los encuentros se sumaron actores del Centro Nacional Patagónico ${ }^{2}$ del CONICET que trabajan en el área de Comunicación de la Ciencia y la Educación, interesados en participar en la organización de las actividades en el barrio. El contacto con estos actores se estableció a partir del taller "Comunicación, Ciencia y Sociedad" desarrollado en el marco del III Encuentro Nacional de Ciencia y Universidad en el mes de octubre de 2018, en el CENPAT y la Universidad Nacional de la Patagonia San Juan Bosco de la sede Puerto Madryn. En el mes de octubre se realizaron de manera conjunta tareas de campo en el

\footnotetext{
${ }^{2}$ En adelante CENPAT
} 
Barrio Nueva Chubut y se entrevistó a la persona referente del sector. Luego de esos primeros encuentros surgieron otros, y actualmente se están delineando tareas conjuntas con dos propósitos principales, por un lado, trabajar colaborativamente con los actores en el territorio y, por otro lado, dar a conocer a la sociedad las problemáticas identificadas en el barrio. Reconociendo el valor del diálogo de saberes (Freire, 1996), el trabajo se aborda aplicando metodologías participativas de investigación, con el objetivo de hacer conversar el saber científico con el saber de los actores en el territorio.

\section{CARACTERIZACIÓN DE LA PROBLEMÁTICA DE ESTUDIO}

Abordar al acceso al hábitat como problemática sociopolítica y territorial, implica reconocer a los procesos de toma de tierras y a la formación de asentamientos informales como una de las tantas maneras en que se materializan las respuestas populares ante los obstáculos que impiden su acceso al suelo urbano. Éste constituye un factor decisivo en la integración a la ciudad y en el derecho a la ciudadanía (Canestraro, 2016). Sin embargo, las experiencias demuestran que para algunos sectores sociales es más bien un elemento de exclusión. Puerto Madryn, ciudad intermedia ubicada al nordeste de la Provincia de Chubut en la Patagonia central argentina (ver figura 1)-, no es ajena a esta realidad. Las desigualdades en el acceso al hábitat en la ciudad se explican como consecuencia de diversos factores. Por un lado, un crecimiento demográfico destacado y sostenido desde 1970 a la actualidad que no ha sido acompañado por una planificación urbana acorde a ese proceso de expansión; y por otro lado, las restricciones y limitaciones que el mercado inmobiliario y el Estado fueron imponiendo a los sectores sociales más desfavorecidos socioeconómicamente en el acceso al suelo, la vivienda y los servicios urbanos. Dichos factores no sólo han promovido procesos de toma de tierras en determinados sectores de la ciudad, sino que además, han profundizado la segregación socio-territorial y con ella, las desigualdades urbanas. 


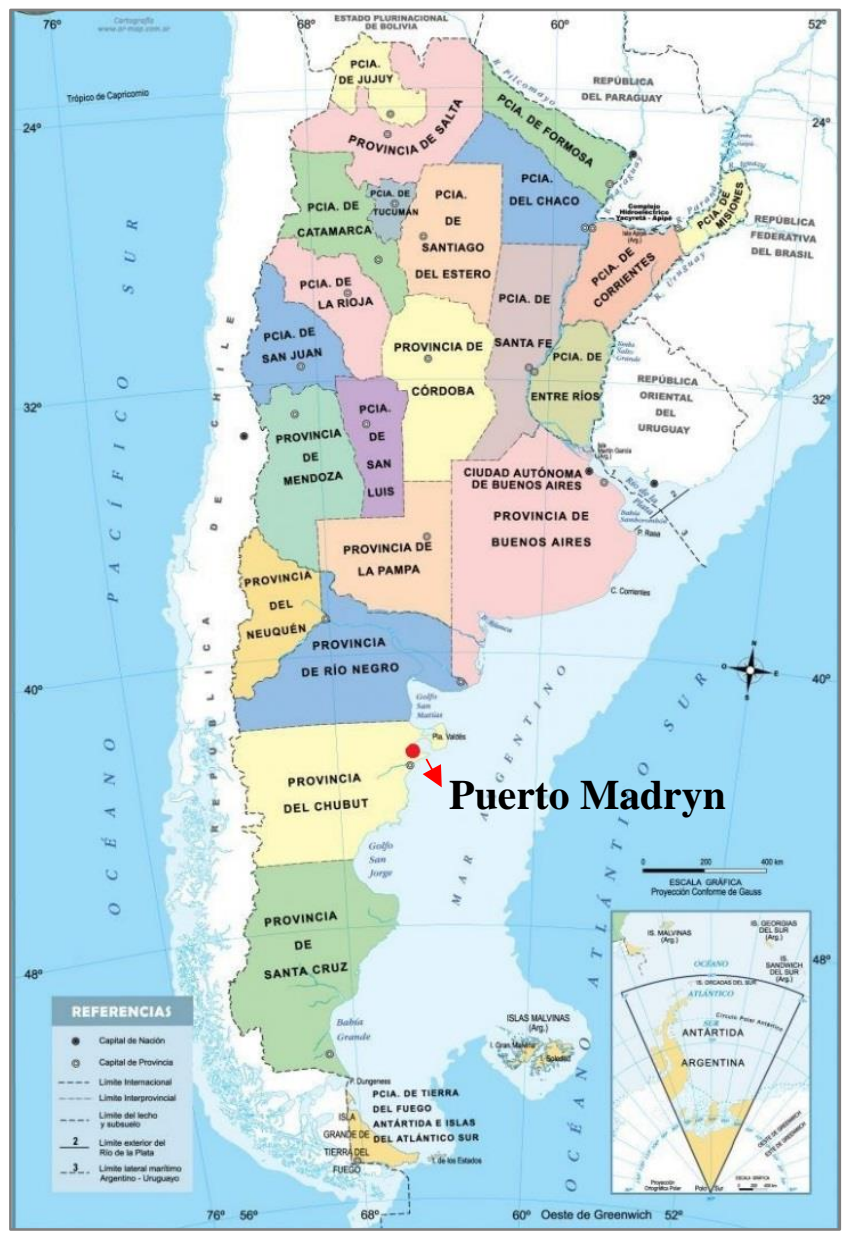

Figura 1. Localización de la ciudad de Puerto Madryn, Patagonia Argentina. Fuente: elaboración propia sobre imagen del Instituto Geográfico Nacional (IGN)

La segregación en el aspecto social, territorial y residencial alude a aquel proceso que mediante diversos mecanismos y prácticas sociales -públicas y del sector privado principalmente- distribuye a diferentes grupos sociales en el territorio, con importantes consecuencias respecto a las posibilidades de acceso a los servicios y los bienes urbanos. En Puerto Madryn el proceso de expansión urbana ha tenido dos etapas bien diferenciadas. Una que va de los '70 a los '90 en la cual el Estado se ha desempeñado como planificador de ese proceso. Y del '90 en adelante se evidencia un proceso de expansión desregulada, en el que las prácticas de distribución y expansión del suelo y la vivienda se descentralizan y aparecen múltiples actores, haciendo más restrictivo el acceso a la residencia para los sectores populares.

El Barrio Nueva Chubut surgió a partir de toma de tierras hacia el año 2003. Las tomas iniciales se registraron en un sector denominado por sus ocupantes como "Nueva Chubut" y posteriormente se incrementaron $\mathrm{y}$ extendieron espacialmente, dando lugar a un conjunto de asentamientos 
autodenominados por sus habitantes: 27 de Octubre, La Arboleda, La Lomita, Nueva Esperanza, Nueva Chubut, Nuevo Madryn y Alta Tensión (Figura 2).

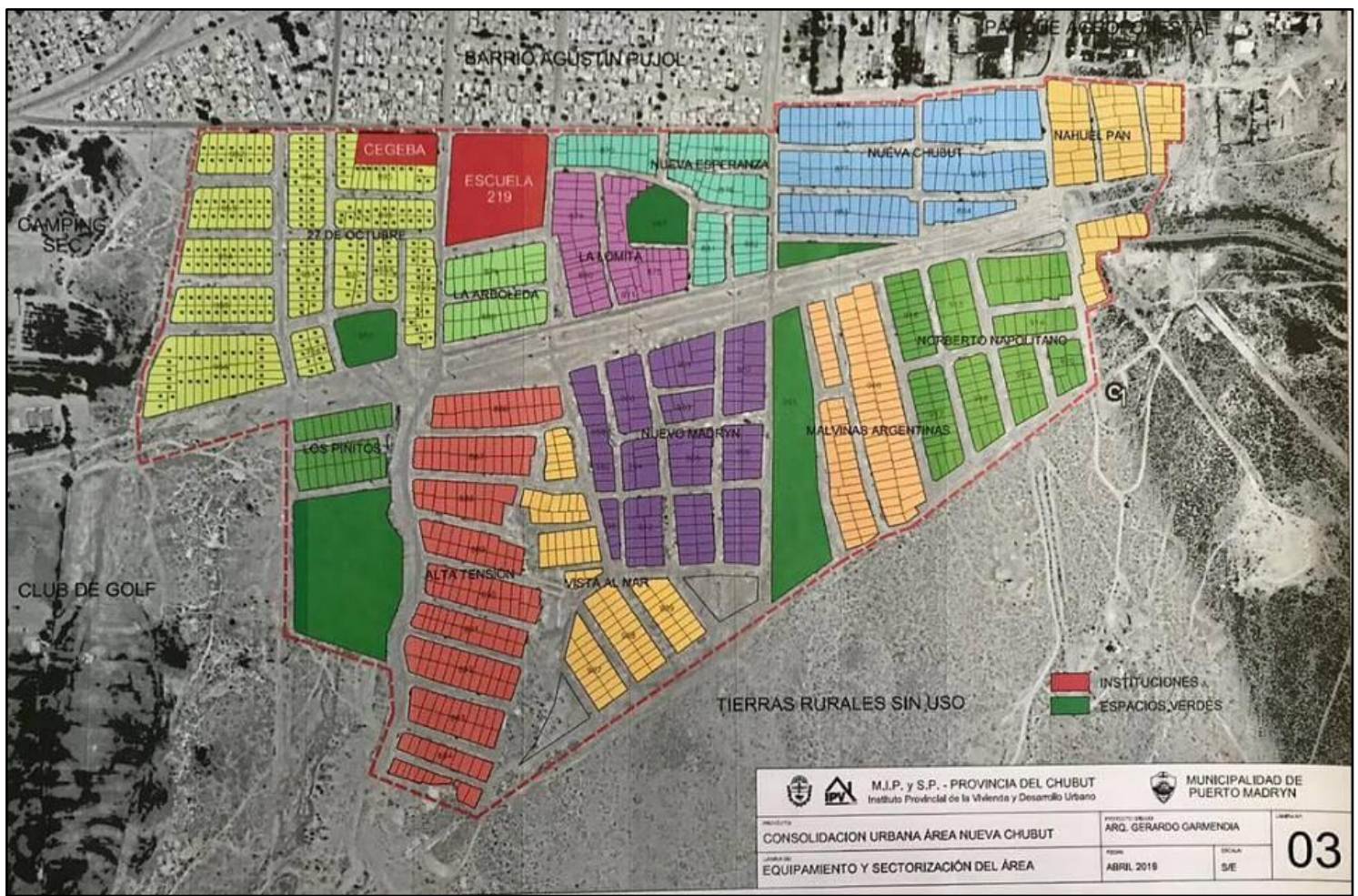

Figura 2. Delimitación del Barrio Nueva Chubut con los asentamientos constitutivos y las nuevas ocupaciones del borde. Fuente: Instituto Provincial de la Vivienda y Desarrollo Urbano, Provincia del Chubut.

Actualmente el sector de estudio se encuentra integrado a la trama urbana de la ciudad de Madryn bajo la denominación de Barrio Nueva Chubut ${ }^{3}$, el cual se encuentra constituido por los asentamientos mencionados anteriormente, más un sector de nuevas ocupaciones de tierra originadas en los años 2017 y 2018. Las mismas corresponden a los asentamientos Malvinas Argentinas, Norberto Napolitano, Nahuel Huapi, Los Pinitos, Vista al Mar y la extensión del asentamiento Nahuel Pan.

Respecto a la organización territorial del sector, se registran dos intervenciones principales: la de la comunidad y la del Estado. Las tomas iniciales se realizaron gracias a la voluntad, persistencia y organización de un grupo de familias que fueron ocupando la tierra, colaborando entre sí en el proceso de autoconstrucción de sus viviendas, monitoreando y advirtiéndose sobre las probables usurpaciones por parte de otras familias, y colaborando en el acceso a las tomas de electricidad y agua (Ferrari, 2018).

\footnotetext{
${ }^{3}$ El nombre fue dado en consideración al primer asentamiento
} 
La ocupación se fue organizando entre las propias familias, que iban siguiendo el trazado de las calles aledañas del barrio contiguo Pujol II. En algunos sectores de ocupación más reciente, como es el caso de los asentamiento Nueva Madryn, Malvinas Argentinas y Norberto Napolitano, hubo acompañamiento por parte del Estado Municipal durante el momento de las tomas, principalmente en lo concerniente a los sitios más aptos para instalarse por sus condiciones topográficas.

Desde la efectivización de las tomas iniciales al día de hoy transcurrieron dieciséis años, período en el que el barrio evidenció un proceso de transformación gradual tanto en el plano material como en las dinámicas organizativas, gestadas por las necesidades y el compromiso de las familias asentadas, quienes fueron defendiendo y reclamando lo que consideraban necesario para su subsistencia. Para la resolución de los problemas, a la comunidad del Barrio Nueva Chubut la interpela la modalidad de resolución mediante la acción y el reclamo a través de las referentes ${ }^{4}$. El trabajo cotidiano y sostenido en procura de mejorar los bienes y servicios de uso común, posiciona a la comunidad en el lugar de decisión sobre su territorio y sus condiciones de reproducción de la vida cotidiana.

\section{MARCO TEÓRICO Y METODOLÓGICO DE REFERENCIA}

\section{La Investigación Acción Participativa como enfoque y metodología}

Un modo de investigación no muy difundido en las instituciones científicas y académicas es la Investigación Acción Participativa (IAP). Este modo de entender la investigación científica da a las comunidades un rol esencial, dado que ellas deben ser partícipes de las distintas fases del proceso. La IAP es un enfoque investigativo y una metodología de investigación que se realiza desde y con la comunidad, donde los/las investigadores/ras se involucran e integran con sus miembros para estudiar los problemas y las posibles soluciones. Desde este enfoque, los problemas a investigar son definidos, analizados y resueltos conjuntamente, con el propósito de que la comunidad vaya siendo autogestora del proceso, apropiándose de él y teniendo un control operativo (saber hacer), lógico (entender) y crítico

\footnotetext{
${ }^{4}$ Quienes ejercen las tareas de canalización de las demandas de las familias y los reclamos ante el Estado son mayoritariamente mujeres.
} 
(juzgar) del propio proceso investigativo (Lewin, 1992). Como enfoque, la IAP aporta una orientación teórica y epistemológica en torno al modo investigar; y como metodología, hace referencia a procedimientos específicos para llevar adelante una investigación. Lo novedoso radica en el sentido e implicancias de los términos acción y participación. No sólo es investigación, sino investigación participativa e investigación-acción; es decir, que involucra la presencia real, concreta y en interrelación de la investigación, de la acción y de la participación.

Es investigación porque se orienta en un proceso de estudio de la realidad o de aspectos determinados de ella con rigor científico. Es acción, entendida como aquella que conduce al cambio social estructural. Para algunos la acción es denominada praxis, es decir, el resultado de la reflexión continua sobre la realidad abordada, no sólo para conocerla sino para transformarla. La acción -solidaria y transformadora- se da durante todo el proceso de la investigación y a la vez va incidiendo sobre la realidad que se investiga. Y por último, es participativa en la medida que en el proceso están involucrados/as no sólo los/as investigadores/as y profesionales, sino los actores de la comunidad que no son considerados/as como objeto de investigación sino como sujetos activos que contribuyen a conocer y transformar su propia realidad.

Se trata de una metodología de trabajo participativo, donde los actores implicados se convierten en protagonistas del proceso de construcción del conocimiento de la realidad objeto de estudio, activos en la detección de problemas y necesidades y en la elaboración de propuestas y soluciones. A los fines de detectar las demandas relacionadas con la problemática de estudio se desarrolla un proceso de investigación que apunta a la transformación mediante el trabajo con colectivos, asociaciones, grupos de vecinos/as y otros actores del municipio con sensibilidades o intereses comunes, lo cual facilita una movilización hacia la implicación ciudadana que favorece la creatividad social en beneficio de toda la comunidad local. El conocimiento de la realidad se construye progresivamente en un proceso participativo en el cual los actores implicados "tienen la palabra" y de este modo se crean las condiciones que facilitan espacios de reflexión, integración e inclusión social en la acción. 
La metodología empleada en las Mesas de Trabajo Permanente se basa en los postulados de la IAP, donde la participación específica y activa de los actores involucrados en el Barrio Nueva Chubut, en las diversas etapas que constituyen a las mismas: su organización, desarrollo y proyecciones; son claves. Del estudio sobre la bibliografía vinculada a las metodologías de investigación-acción participativas, se reconoce en las mismas una sólida propuesta de investigar "para y desde la acción" con los actores sociales protagonistas (Paño Yáñez et al, 2019).

\section{Una aproximación al territorio y su vinculación con las metodologías participativas}

La extensa conceptualización referida al territorio en el campo de estudio de la geografía da cuenta de una amplia producción desde diversos enfoques teórico-epistemológicos que abordan el concepto, ejemplificada en las explicaciones basadas en entender al territorio como producto de las relaciones entre los binomios sociedad-naturaleza, cultura-naturaleza, ambiente-sociedad; incluida la conceptualización propuesta por Santos (2000) referida al espacio como "naturaleza humanizada". No obstante, en el abordaje de cualquier problemática vinculada al territorio es necesario reconocer la relevancia de éste con el ejercicio del poder. En este sentido, el territorio podría definirse como aquella porción del espaciotiempo en la cual un individuo o grupo intenta ejercer el control sobre determinados procesos, fenómenos y relaciones; es decir, el territorio es entendido desde esta concepción como producto social construido y como proceso histórico, en línea con la concepción lefrebvriana.

La territorialización como proceso que organiza y construye territorios abarca relaciones de poder, representaciones y acciones que establecen grupos sociales para estructurar el espacio (Hensler et al, 2019). Esta mirada conceptual permite visibilizar los modos en que diferentes racionalidades e intereses organizan el territorio, siendo éste un reflejo de la construcción histórica. Como señala Porto-Gonçalves (2001), es necesario "desnaturalizar el territorio y reconocer procesos de territorialización” (2001:17). En este sentido, Albaladejo (2004) -basándose en la teoría de las transformaciones territoriales de Raffestin (1987)- introduce una visión dinámica del concepto de territorio. Desde esta teoría el territorio es entendido como un conjunto de vínculos conceptuales y materiales entre las sociedades y sus espacios, que experimenta continuas transformaciones, y por lo tanto, procesos de construcción de territorios 
nuevos (reterritorialización) con la correlativa destrucción del orden territorial anterior (desterritorialización). De esta manera, las transformaciones territoriales se constituyen en "procesos permanentes de degradación y de reconstrucción del territorio" (Carricart y Albaladejo, 2005: 4).

Abordar cualquier problemática desde un enfoque cuyo eje sea el territorio, da a los contextos sociales, económicos, políticos y materiales en los cuales están insertas las comunidades estudiadas un rol central, a partir el cual es factible delinear estrategias de desarrollo (Schneider y Peyré Tartaruga, 2006). El enfoque territorial es de gran utilidad en la medida que permite abordar de manera integral las realidades de una comunidad, y a la vez, diseñar estrategias participativas de resolución de las problemáticas identificadas, contribuyendo al "empoderamiento por parte de los/as líderes comunales, porque son estos/as los/as que deciden el uso que se le debe dar a sus recursos y, por ende, a su territorio" (Alvarado Sánchez y Flores Abogabir, 2011: 214).

En las tradicionales formas de realizar investigación y extensión desde las universidades subyace una modalidad en relación con los territorios, donde existe un sector que detenta el saber (las universidades y los organismos de ciencia y tecnología) y se lo transfieren a otro que lo ignora (la sociedad). Desde la perspectiva del desarrollo territorial participativo se entiende que hay diversos tipos de conocimientos y que ninguno es superior a los otros. Desde este contexto, el conocimiento académico debe combinarse también con otros tipos de saberes presentes en el territorio en búsqueda de soluciones compartidas. Esto cambia la manera de involucrarse en el territorio y obliga a una reflexión sobre cómo evolucionan los procesos en estos espacios.

Las Mesas de Trabajo del Barrio Nueva Chubut - encaradas desde las bases propuestas por los enfoque de la IAP y territorial- se encuentra en un estadío inicial y exploratorio dado el tiempo de vigencia e implementación del mecanismo mencionado; si bien sustentado en el abordaje de experiencias de participación orientadas a la transformación del territorio de estudio. Lo que se propone desde las mesas de trabajo es la participación comunitaria que atienda las problemáticas territoriales más cercanas, en el corto plazo y asociadas al barrio y su gente. Asimismo, en el trabajo participativo es clave delimitar la escala. El barrio, como escala micro-local, por su tamaño territorial y poblacional, en general es visto 
como un ámbito propicio para la participación, por la mayor identificación y sentido de pertenencia ciudadana (Signorelli, 2019). En este sentido, la escala -categoría de análisis de la geografía como disciplina científica- aporta un sentido político al territorio, entendido como espacio de relaciones sociales y como tal, social e históricamente producido (Lefevbre, 2013).

El propósito es que ciudadanos y ciudadanas encuentren canales de participación, expresión e intervención conjunta para atender problemas concretos que los/as afectan diariamente. Los procesos de gestión compartida del territorio son cada vez más visibilizados desde algunas prácticas investigativas, los mismos "incluyen o aspiran a una amplia participación social, donde la diversidad de visiones, el diálogo de saberes y la acción colectiva permitan mantener o generar formas de vida más sustentables y justas, con mayor paz social y territorial" (Hensler et al, 2019: 237). Estos procesos de cogestión del territorio implican una transformación individual y colectiva que se construye en el diálogo y la reflexión sobre nuestras prácticas. La articulación entre organizaciones de la sociedad civil, la academia, la ciencia y miembros de diferentes comunidades representa un logro clave para el fortalecimiento de las iniciativas locales orientadas a construir territorios más justos e igualitarios.

\section{LA MESA DE TRABAJO DEL BARRIO NUEVA CHUBUT: EXPERIENCIAS Y PRIMEROS RESULTADOS}

\section{Inicio del trabajo de campo en el territorio y con los actores}

Durante los primeros meses de llegada al territorio las tareas estuvieron orientadas a realizar un relevamiento socio-cultural del sector. Se recorrió gran parte de las calles del Barrio Nueva Chubut, conversando con vecinos/as de los distintos asentamientos y contactando a las personas referentes de cada sector. En algunos momentos realizando entrevistas en profundidad y en otros, encuestas personales para conocer sus procedencias e historias en el barrio, así como su relación con el entorno, con las familias vecinas, sus preocupaciones e intereses. Asimismo, se estableció el contacto con actores del Estado Municipal y Provincial encargados de realizar tareas y ejecutar programas de mejoramiento de las condiciones habitacionales y sociales del barrio. El resultado de esta etapa diagnóstica fue la 
elaboración de un mapa de actores e instituciones que estaban trabajando en el territorio (ver tabla 1). El mapa de actores proporcionó una visión interesante de las relaciones políticas en el barrio y de las demandas y acciones colectivas que los actores llevaban adelante. Además, posibilitó el contacto con las personas referentes con las que se inició la experiencia de organización y participación comunitaria en la mesa de trabajo. La propuesta fue trabajar juntos en la conformación de un espacio de encuentro, de deliberación y de concertación para los asuntos comunes.

\begin{tabular}{|c|c|c|}
\hline \multicolumn{2}{|c|}{ Tipología de actores } & Actores \\
\hline \multirow[t]{2}{*}{$\begin{array}{l}\text { Sector } \\
\text { público }\end{array}$} & Municipal & $\begin{array}{l}\text { - Subsecretaría de Ecología y Protección Ambiental } \\
\text { - Secretaría de Desarrollo Urbano } \\
\text { - Secretaría de Acción Social y Desarrollo Comunitario } \\
\text { - Dirección Municipal de Tierras } \\
\text { - Centro de Gestión Barrial }\end{array}$ \\
\hline & Provincial & - Instituto Provincial de la Vivienda y Desarrollo Urbano \\
\hline \multicolumn{2}{|c|}{$\begin{array}{l}\text { Sector } \\
\text { privado/empresarial }\end{array}$} & $\begin{array}{l}\text { - Empresa Fabri (constructora encargada de ejecutar las obras en el } \\
\text { marco del Programa de Mejoramiento de Barrios - PROMEBA) }\end{array}$ \\
\hline \multicolumn{2}{|c|}{$\begin{array}{l}\text { Sector científico- } \\
\text { académico }\end{array}$} & $\begin{array}{l}\text { - Centro Nacional Patagónico -Consejo Nacional de Investigaciones } \\
\text { Científicas y Técnicas - CONICET } \\
\text { - Universidad Nacional de la Patagonia San Juan Bosco }\end{array}$ \\
\hline \multicolumn{2}{|c|}{$\begin{array}{l}\text { Organizaciones de la } \\
\text { sociedad civil y políticas }\end{array}$} & $\begin{array}{l}\text { - Asociación Autónoma Cascos Blancos } \\
\text { - Barrios de Pie } \\
\text { - La Garganta Poderosa } \\
\text { - Unión y Organización por Chubut }\end{array}$ \\
\hline \multicolumn{2}{|c|}{ Comunidad barrial } & $\begin{array}{l}\text { - Referentes de los asentamientos Nueva Madryn, Alta Tensión, La } \\
\text { Lomita, } 27 \text { de Octubre y La Arboleda. } \\
\text { - Vecinos y vecinas }\end{array}$ \\
\hline
\end{tabular}

Tabla 1. Mapa de actores del Barrio Nueva Chubut. Fuente: elaboración propia.

\section{Los primeros pasos de la investigación-acción participativa desde la Mesa}

El trabajo de organización y realización de la Mesa de Trabajo del Barrio Nueva Chubut involucró diversas etapas. En una primera etapa se realizaron los contactos iniciales con las personas referentes del sector interesadas en promover el involucramiento de las/os vecinas/os en la gestión de sus recursos mediante el uso de metodologías participativas. En esta primera instancia participaron referentes del sector, vecinas y vecinos, actores del CONICET-CENPAT y asociaciones civiles, con quienes establecimos los objetivos y acuerdos generales de trabajo, los compromisos a asumir por los diferentes actores involucrados, los recursos con los que se disponía, entre otros. En el mes de octubre de 2018 se 
organizaron varios encuentros con el propósito de construir los primeros lazos y delinear las primeras actividades.

En una segunda etapa se apuntó a identificar las problemáticas sobre las cuales trabajar. Así en los meses de enero y febrero de 2019 se comenzó a organizar una serie de encuentros a los que se invitó a la comunidad en general y a referentes institucionales. La idea propuesta fue identificar un conjunto de problemáticas a partir de las cuales construir un proyecto comunitario que promoviera procesos participativos y organizativos. De dichos encuentros surgieron diversas preocupaciones prioritarias y posibles actividades a realizar para su resolución. Las mismas se detallan en la tabla 2.

\begin{tabular}{l|l}
\hline \multicolumn{1}{c|}{ Problemas identificados } & \multicolumn{1}{c}{ Posibles soluciones } \\
\hline $\begin{array}{l}\text { Adicciones en grupos de } \\
\text { adolescentes }\end{array}$ & $\begin{array}{l}\text { Modos de recuperación y reinserción laboral: actividades de huerta } \\
\text { comunitaria, asistencia en talleres de oficios. }\end{array}$ \\
\hline $\begin{array}{l}\text { Alcoholismo en mujeres y } \\
\text { hombres }\end{array}$ & $\begin{array}{l}\text { Acompañamiento de los casos mediante equipos interdisciplinarios } \\
\text { y con instituciones del Estado. }\end{array}$ \\
\hline $\begin{array}{l}\text { Situaciones de violencia de } \\
\text { género }\end{array}$ & $\begin{array}{l}\text { Estimular a las mujeres el barrio para que hagan las denuncias } \\
\text { (charlas y/o talleres) y sean acompañadas durante el proceso de }\end{array}$ \\
& $\begin{array}{l}\text { seguimiento y contención ante un caso de violencia en el contexto } \\
\text { familiar. }\end{array}$ \\
\hline $\begin{array}{l}\text { Niñas y niños sin } \\
\text { contención familiar }\end{array}$ & $\begin{array}{l}\text { Generar espacios de contención y acompañamiento mediante } \\
\text { actividades de asistencia en merenderos comunitarios, clases de }\end{array}$ \\
apoyo escolar u otra actividad; dada la presencia de niños/as en \\
espacios públicos sin acompañamiento familiar durante horas \\
prolongadas, al igual que en los contextos familiares.
\end{tabular}

Tabla 2. Resultados Mesa de Trabajo. Fuente: elaboración propia.

En una tercera etapa, considerando el amplio abanico de problemáticas enunciadas por los/as vecinos/as del barrio, se procedió a establecer un orden de prioridades sobre las cuales comenzar a trabajar. Se distribuyeron tareas a los efectos de profundizar en la precisión de las acciones en términos de calidad, tiempos, financiamiento y responsabilidades. Los participantes definieron en intercambios colectivos, qué se va a hacer, cómo, con quién, con qué recursos, en qué plazos y con qué propósito. 


\section{Las actividades materializadas en compromisos asumidos}

En el mes de febrero de 2019 se organizó una salida al Parque de Diversiones de Puerto Madryn con familias, niñas y niños del barrio Nueva Chubut. Ante la gestión de entradas gratuitas por parte de una vecina, la dificultad que se presentó inicialmente fue la carencia de movilidad que pudiera trasladar a todos/as hacia el predio del parque. En una primera instancia se intentó conseguir un colectivo para el traslado, y por dificultades presupuestarias se decidió -entre los actores de la mesa- aportar acciones solidarias entre vecinos y vecinas del barrio y actores del CENPAT para disponer de vehículos particulares y poder acercar a las familias hasta el parque. Asistieron aproximadamente unas 40 a 50 niñas y niños (figura 3).

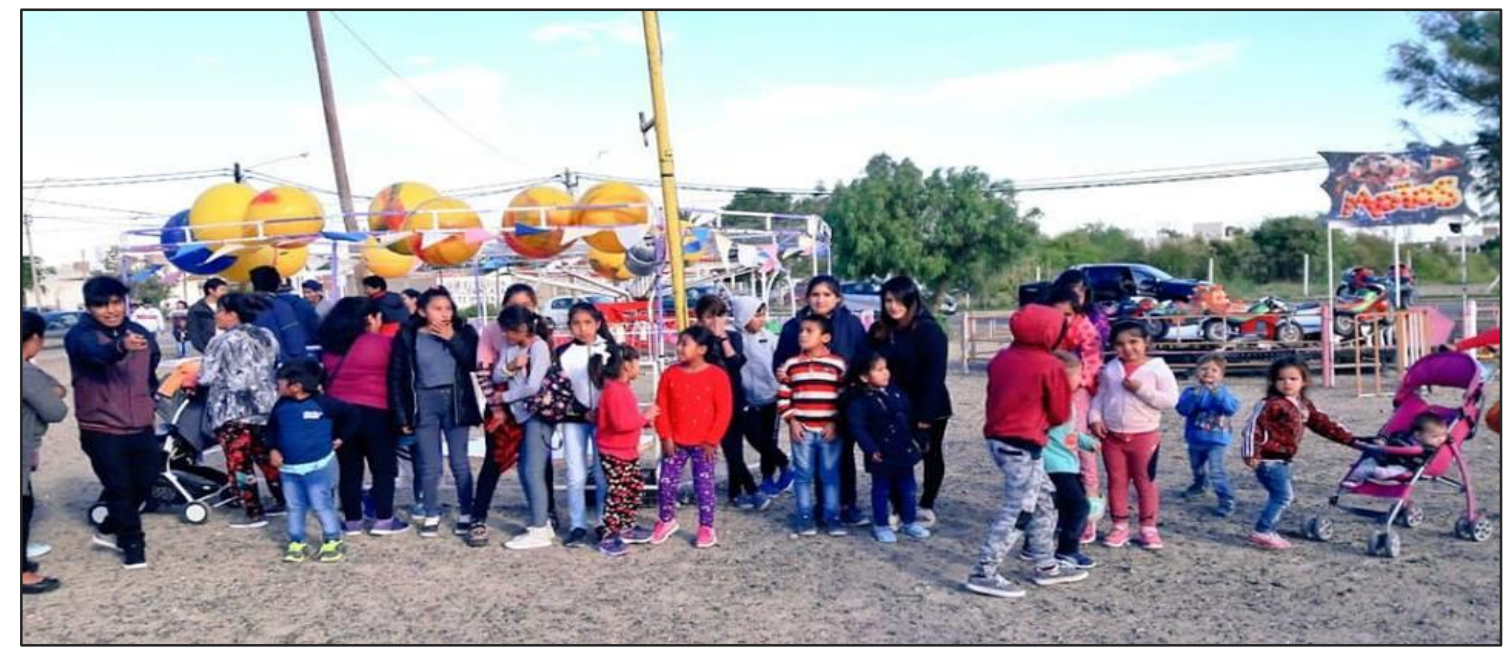

Figura 3. Salida al Parque de Diversiones con familias el Barrio Nueva Chubut. Fuente: registro propio, fecha 10/02/2019, Puerto Madryn.

El día 27 de febrero de 2019 se concretó otra Mesa de Trabajo con actores de la comunidad ${ }^{5}$, del CONICET-CENPAT ${ }^{6}$ y de la Asociación Autónoma Cascos Blancos ${ }^{7}$ La metodología con la cual se trabajó en el encuentro se basó en escuchar las inquietudes de los/as vecinos/as del barrio, con el propósito de identificar las problemáticas prioritarias a atender. Algunos de los interrogantes disparadores fueron: ¿Cuáles son las necesidades urgentes a atender en el barrio? ¿Qué pueden hacer -

\footnotetext{
${ }^{5}$ Isabel Morrison (referente del Barrio), vecinos y vecinas

${ }^{6}$ Ciencia y Comunicación: Diego Nuñez de la Rosa, Alejandro Cannizzaro, Ciencia y Educación: Irupé Carmona; Instituto Patagónico de Ciencias Sociales y Humanas CONICET: Anahí Ruderman, Paula Ferrari.

${ }^{7}$ es una asociación autónoma de Italia que trabaja en el barrio desde hace unos años acompañando a adolescentes con problemas de adicción, orientándolos con diversas actividades: clases de apoyo, trabajo en huertas y trabajo voluntario.
} 
los actores presentes- para colaborar con los problemas y/o necesidades del barrio? ¿Qué actividades pueden desplegarse para dar solución a las problemáticas prioritarias a atender?

Ante la identificación de la dispersión de basura como problemática urgente que afecta a las familias del Barrio Nueva Chubut, el compromiso asumido entre los actores presentes en la Mesa de Trabajo fue colaborar en las siguientes tareas: Recorrer el barrio hablando con los/as vecinos/as sobre la importancia de mantener el lugar en buenas condiciones de higiene y limpieza. Mediante la entrega de folletería informativa o bien mediante charlas referidas al tema. Las figuras 4 y 5 corresponden a las reuniones mantenidas en la Mesa de Trabajo del día 27 de febrero.

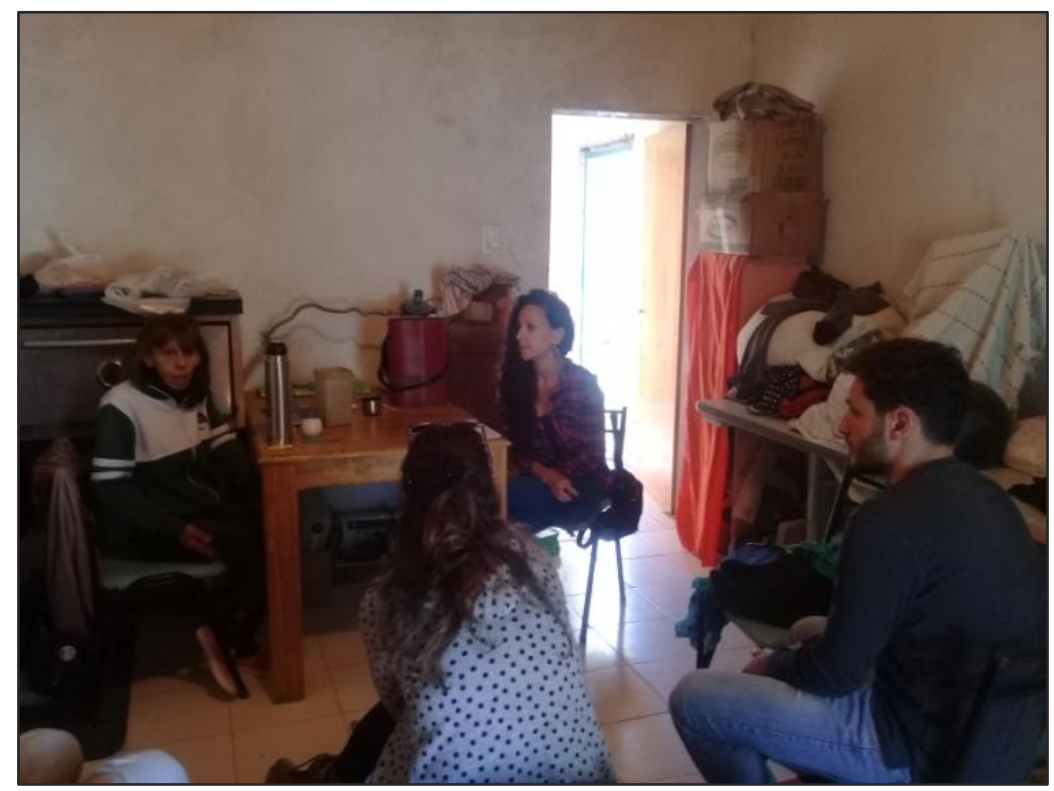

Figura 4. Mesa de Trabajo realizada en el mes de febrero de 2019. Actores en la imagen: referente barrial, del CenpatConicet y Asociación Civil Cascos Blancos. Fuente: registro propio. 


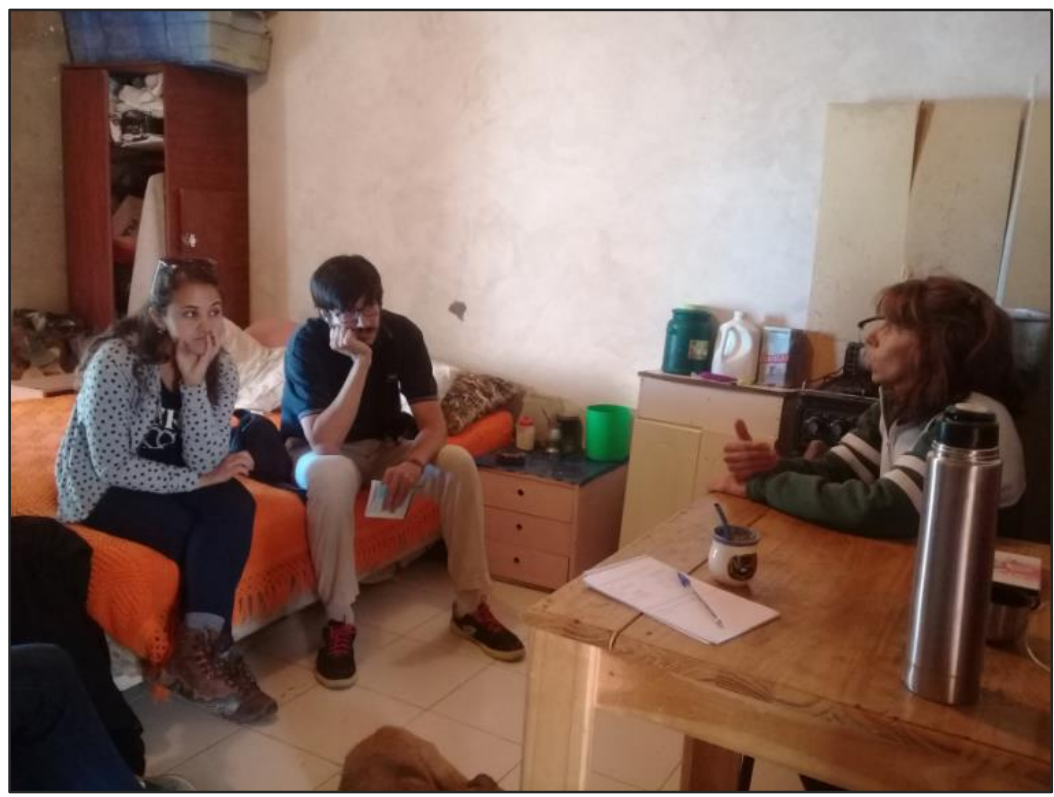

Figura 4. Mesa de Trabajo del mes de febrero de 2019. Lugar de realización: vivienda de la referente barrial (Isabel Morrison). Actores en la imagen: referente barrial y del Cenpat-Conicet. Fuente: registro propio.

Fuente: registro propio, fecha 10/02/2019, Puerto Madryn.

A su vez, actualmente se está organizando un taller de material reciclado para la confección de cestos de basura. Con la colaboración de un artista plástico que reside en la ciudad de Puerto Madryn dedicado a esa tarea, se mantuvieron reuniones para delinear y definir la modalidad del taller, los insumos necesarios, el momento y lugar donde se llevará a cabo, a los fines de hacer una convocatoria amplia entre las familias del barrio.

\section{REFLEXIONES FINALES}

Se parte de reconocer al proceso investigativo no sólo basado en acciones conjuntas desde el primer momento, sino en la participación de la comunidad involucrada. Este posicionamiento se aleja de entender a la investigación al servicio de unos pocos, como habitualmente es practicada desde contextos académico-universitarios. Por el contrario, se trata de un modo de entendimiento y aplicación de la investigación y la ciencia al servicio de la colectividad; aportando a la resolución de sus problemas y necesidades.

En este sentido, las mesas de Trabajo basadas en procedimientos aportados por la investigación-acción participativa, logran introducir otro modo de hacer investigación, y así deconstruir la concepción y las practicas del proceso de investigación predominante en las instituciones científico-académicas. El 
ejercicio de la participación implica analizar y cuestionar las prácticas cotidianas que reproducen situaciones de dominación, y adquirir nuevas herramientas para la gestión democrática de los recursos existentes en cada territorio. En este sentido, se busca fomentar la construcción de espacios de participación que obliguen a revisar las estructuras y formas organizativas que atraviesan y pautan el modo de relacionamiento entre los actores que trabajan en cada comunidad, donde el Estado no es el único protagonista. Esto implica poner especial atención a la forma en que los actores asumen responsabilidades en la toma de decisiones de la gestión de la política pública a partir de los espacios de mediación entre el Estado y la sociedad civil.

Asimismo, se reconoce a las diversas instancias de gobierno del Estado municipal y/o provincial partícipes necesarios y responsables en la cogestión de las acciones de mejoramiento y transformación de las condiciones de vida y habitacionales de las familias del barrio, encaradas desde la mesa de trabajo. No obstante, al momento de organizar los primeros encuentros de la mesa del Barrio Nueva Chubut, se iniciaba la campaña política para la elección de representantes municipales en la ciudad de Puerto Madryn y, por lo tanto, los actores de gobierno responsables de diversos programas, secretarías y centros de gestión barrial, se encontraban en pleno desarrollo de actividades partidarias y de campaña electoral; situación que llevó a decidir, entre los actores participes de la mesas iniciales (científico-académicos, comunidad y asociación civil) no convocarlos a esos encuentros, dada la primacía de sus intereses electorales y partidarios, que introdujeron complejidades y tergiversaciones en algunas de las actividades llevadas a cabo al inicio.

A su vez, la intervención del sector científico-académico en la mesa de trabajo del Barrio Nueva Chubut es participativa en la medida en que ese colectivo es parte de la mesa y en este sentido construye su propia práctica junto a la comunidad del barrio. Se reconoce a los/as vecinos/as como sujetos políticos con capitales y conocimientos que les permite desenvolverse en el espacio donde desarrollan cotidianamente sus prácticas, y además, capaces de reflexionar y dar sentido a sus prácticas desde una perspectiva, que enriquece y resignifica la de la academia.

\section{REFERENCIAS}


Albaladejo C. (2004). "Innovaciones discretas y reterritorialización de la actividad agropecuaria en Argentina, Brasil y Francia (trad. Isabelle Garma-Berman)". In: Albaladejo C. y Bustos Cara R. (eds.), Desarrollo local y nuevas ruralidades en Argentina. UNS Departamento de Geografía / IRD UR102 / INRA SAD / Univ. Toulouse Le Mirail UMR Dynamiques Rurales, Bahía Blanca, Argentina. p.369- 412.

Alvarado Sánchez, M. y Flores Abogabir, M. (2011) El enfoque territorial en proyectos de extensión universitaria: casos de proyectos en turismo rural comunitario. Revista Geográfica de América Central, Número Especial, p. 213-230.

Canestraro, M.L. (2016) Sobre el derecho a la ciudad y el acceso al suelo urbano. Reflexiones a partir de intervenciones estatales recientes (Mar del Plata, 2012-2015). Estudios Socioterritoriales. Revista de Geografía. N²0, p. 57-74.

Carricart, E.P; Albaladejo, C. (2005) "Reflexiones críticas sobre los espacios emergentes agropecuarios y los espacios rurales en la región pampeana argentina” (49-88 p) En Benencia R. y Flood, C. (ed.) Trayectorias y Contextos. Organizaciones rurales en la Argentina de los noventa. ISBN 987-9028-56-2. Editorial La Colmena. Buenos Aires.

Ferrari, M.P. (2018). Un lugar para vivir: la toma de tierras en el asentamiento informal Nueva Madryn, Patagonia Argentina. Geografía em Questao. Revista de la Asociación de Geógrafos Brasileros. Volumen 11, N², p.23-40.

Freire, P. 1996. Pedagogia da Autonomia. Río de Janeiro: Paz e Terra.

Hensler, L.; Merçon, J.; Estrada Paulin, I.; González-González, R.; Paradowska, K.; Bravo Reyes, L.; Cesareo Lopez, V. (2019) Metodologías participativas para la cogestión del territorio. Una experiencia de aprendizaje colectivo en Veracruz, México. Cap. 12 (p. 235-260) en Paño Yañez et al Comps. Procesos y Metodologías Participativas. Reflexiones y experiencias para la transformación social. Editorial: CLACSO, UDELAR. 1era Edición. Uruguay.

Lefebvre, H. (2013) [1974]. La producción del espacio Capitán Swing. Madrid.

Lewin, K. (1992), "La investigación-acción y los problemas de las minorias", en AA.VV., La investigación-acción participativa. Inicio y desarrollo, Biblioteca de Educación de Adultos, nº 6, Ed. Popular, Madrid, pp. 13-25.

Paño Yáñez, P.; Rébola, R.; Suárez Elías, M. Comps. (2019) Procesos y Metodologías Participativas. Reflexiones y experiencias para la transformación social. Editorial: CLACSO, UDELAR. 1era Edición. Uruguay.

Porto-Gonçalves, C.W. (2001). Geo-grafías. Movimientos sociales, nuevas territorialidades y sustentabilidad (edición en español). México: Siglo XXI.

Raffestin, C. (1987). "Repères pour une théorie de la territorialité humaine". Cahier du Groupe Réseaux, n 7, (263-279 p).

Santos, M. (2000) La naturaleza del Espacio. Técnica y tiempo. Razón y emoción. Editorial Ariel Geografía. España.

Schneider, S. y Peyré Tartaruga, I. (2006). Territorio y Enfoque Territorial: de las referencias cognitivas a los aportes aplicados al análisis de los procesos sociales rurales (71-102) en: MANZANAL, Mabel; NEIMAN, Guillermo y LATTUADA, Mario. (Org.). Desarrollo Rural. Organizaciones, Instituciones y Territorio. Buenos Aires: Editorial Ciccus.

Signorelli, G. (2019) Los desafíos de la participación ciudadana a pequeña escala dada la proximidad como principio de legitimidad del lazo representativo. Asambleas Ciudadanas y Consejo de Seguridad de Vecinos en Pueblo Andino, 2018 (Argentina), cap. 10 (p.198-208) en Paño Yañez et al Comps. Procesos y Metodologías Participativas. Reflexiones y experiencias para la transformación social. Editorial: CLACSO, UDELAR. 1era Edición. Uruguay. 